\title{
Identifying and acting on potentially inappropriate care
}

\section{Study question}

To develop a model to measure potentially inappropriate care in Australian hospitals.

\section{Methods}

Analysis of computerised hospital discharge data $(n=8720$ 771) for all patients in Australian hospitals for the financial year 2010-11. Main outcome measures were hospital-specific incidence of selected diagnosis-procedure pairs (labelled as "do not do" treatments) identified as inappropriate in other literature or by authoritative bodies such as the National Institute for Health and Care Excellence in England (vertebroplasty for painful osteoporotic vertebral fractures, arthroscopic lavage or debridement for osteoarthritis of the knee, laparoscopic uterine nerve ablation for chronic pelvic pain, removing healthy ovaries during a hysterectomy, and hyperbaric oxygen therapy for a range of conditions).

\section{Findings}

Stephen J Duckett DSC, FASSA, FAHMS

Peter Breadon MPA, MPP, BA

Danielle Romanes BA(Hons)

Grattan Institute, Melbourne, VIC.

stephen.duckett@ grattan.edu.au

This is a research article summary. The full-length article is available on mja.com.au: doi: 10.5694/mjal5.00025

In 2010-11, there were 4659 cases of hyperbaric oxygen therapy treatments, 813 cases of knee arthroscopy, 302 cases of vertebroplasty, 79 cases of ovary removal and 35 cases of nerve ablation that were potentially inappropriate. These treatments happened in all states and in public and private hospitals. Some hospitals had markedly higher rates than others (Box). of the identified treatments may reflect coding errors or be justified by a rare combination of patient characteristics.

\section{What this study adds to current knowledge}

This study is the first attempt to quantify inappropriate care in Australian hospitals for a range of conditions, using routine data. Importantly, the procedures have either been shown in academic studies to be inappropriate or are recommended against in guidelines, or both.

\section{Proportion of relevant patients receiving a do-not-do treatment in} public hospitals with capacity to perform the treatment, 2010-11

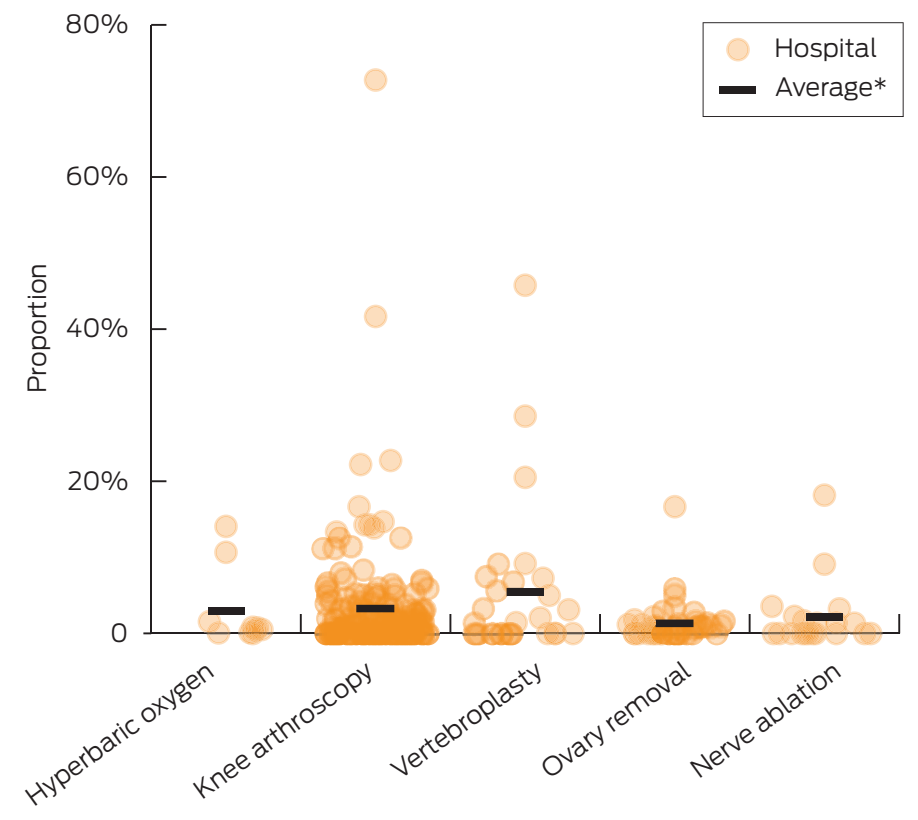

Treatment

\section{Limitations \\ This study only identifies poten- tially inappropriate care. Some}

\section{Implications for practice}

Current strategies are not doing enough. Hospitals should be informed of their relative rates of potentially inappropriate care. If hospitals with unusually high rates do not improve, state health departments should initiate a clinical review of relevant practices. If the review does not endorse clinical practices, and if these practices still do not change, financial sanctions may be required.

Competing interests: No relevant disclosures.

C 2015 AMPCo Pty Ltd. Produced with Elsevier B.V. All rights reserved.

* Average rates among comparator hospitals: hyperbaric oxygen therapy (2.9\%); arthroscopic lavage or debridement of the knee (3.3\%); vertebroplasty $(5.4 \%)$; oophorectomy $(1.4 \%)$; uterine nerve ablation $(2.1 \%)$. 


\section{Identifying and acting on potentially inappropriate care}

I dentifying when the wrong treatments are chosen, and putting a stop to it, is an important way to improve the quality of health care. There are many ways to do this. Strategies include using clinical guidelines or decision support systems, clinician education, clinical engagement, peer review, and adjusting the pricing, funding and availability of individual treatments. All of these methods can be useful. But there are currently few concerted efforts to evaluate and benchmark treatment choices at the hospital level, and to use this information to drive improvement.

Inappropriate care is a longstanding concern in health policy. For over 40 years, small-area analyses have shown significant geographic variation in the rates at which different subpopulations are given common surgical procedures. ${ }^{1,2}$ The Organisation for Economic Co-operation and Development has recently published this kind of analysis, including a chapter on Australia showing the rates of nine types of hospital admission in different Medicare Local areas, adjusted for age and sex. ${ }^{3}$ A more detailed atlas of variation and supporting studies has also been published by the Australian Commission on Safety and Quality in Health Care. ${ }^{4}$

Wide variation in practice patterns has been attributed to clinicians interpreting evidence and guidelines inconsistently, with patients in different areas being under- and overtreated as a result.

Stephen J Duckett DSC, FASSA, FAHMS

Peter Breadon MPA, MPP, BA

Danielle Romanes BA(Hons)

Grattan Institute, Melbourne, VIC

stephen.duckett@ grattan.edu.au

\section{Abstract}

Objective: To develop a model to measure potentially inappropriate care in Australian hospitals.

Design: Secondary analysis of computerised hospital discharge data for all Australian hospitals for the 2010-11 financial year.

Main outcome measure: Hospital-specific incidence of selected diagnosis-procedure pairs identified as inappropriate in other literature.

Results: Five hospital procedures that are not supported by clinical evidence happened more than 100 times a week, on average. The most frequent of these do-not-do treatments was hyperbaric oxygen therapy for a range of specific conditions (4659 admissions in 2010-11). The rate of donot-do procedures varied greatly, even among comparator hospitals that provided the procedure and that treated the relevant patient group. Among comparator hospitals, an average of $3.3 \%$ of patients with osteoarthritis of the knee received arthroscopic lavage and debridement of the knee (a do-not-do treatment), but four hospitals had rates of over $20 \%$. There was also great variation in hospital-specific rates of procedures that should not be done routinely.

Conclusion: Hospital-specific rates of do-not-do treatments vary greatly. Hospitals should be informed about their relative performance. Hospitals that have sustained, high rates of do-not-do treatments should be subject to external clinical review by expert peers.

Both of these approaches to inappropriate care have shortcomings. Small-area variation analysis has uncovered disturbing variation in patterns of care, but has not produced meaningful policy or practice change. ${ }^{5}$ This is probably owing to the fact that variation analyses rarely take into account legitimate drivers of clinical variation such as patient morbidity and patient preferences, and so are unable to convincingly differentiate warranted from unwarranted variation..$^{6,7}$

Although disinvestment work has produced much more compelling evidence of inappropriate care, it has struggled to achieve meaningful policy change. As this study shows, clinicians can continue to use a treatment long after it has been declared inappropriate.

There are few interventions that are ineffective for all patients and indications. Typically, the value of a treatment varies for different types of patients. This complicates measurement of inappropriate care and the development of policies to reduce it. Across-the-board funding cuts, or even funding cuts among specific subgroups of patients, may ignore clinical heterogeneity and deny funding for valuable, as well as ineffective, care. ${ }^{8}$ A patient may have characteristics that typically rule out a treatment, but have other characteristics that mean they are not well represented in clinical trial samples, or are not eligible for treatments that are recommended as more effective. Approaches are emerging that may help health care organisations and clinicians to distinguish, at the site of care, between patients for whom a certain treatment is warranted and those for whom it is not. ${ }^{8}$ But in many cases the coded data do not capture all of the relevant clinical variation. This presents a serious challenge to measuring and comparing clinical choices in different regions or among providers.

We sought to develop a practical way to identify which hospitals are most likely to be choosing inappropriate 
treatments. Our methods draw on the analytical strengths of variation and disinvestment analysis. From disinvestment analysis, we took a selection of treatments that evidence clearly shows should not be done routinely, or at all. From variation analysis, we focused on outlier hospitals - those providing a "do not do" or "do not do routinely" treatment at rates that are far in excess of the national average.

This approach overcomes several key deficiencies of variation and disinvestment research. First, focusing on procedures listed for disinvestment means that high usage rates can be convincingly linked to inappropriate practice. This is not the case in variation analysis, where the procedures analysed are generally considered effective, and where high relative usage in one area can be due to underprovision in other areas.

Second, using variation to focus on providers with high rates of potentially inappropriate care is a practical way to pursue disinvestment. Rather than advocating the removal of clinician discretion with respect to the procedures analysed, this approach advocates monitoring, with priority given to the providers where care is clearly out of step with both clinical evidence and standard practices, and therefore very likely to be inappropriate.

Third, focusing on hospitals rather than geographic areas allowed us to correct for several major deficiencies in other variation analyses. Focusing on hospitals (and specific specialties within those hospitals) allows for analysis of microcultures of care, which are likely to be obscured when practice patterns are aggregated to a regional level. Further, geographically aggregated analyses generally only make crude adjustments for patient morbidity differences, while our use of rich patient-level data allowed us to correct for variation in morbidity to a much greater extent. Finally, there are few viable policy options for dealing with variation at the geographic level. Developing strategies aimed at hospital-level accountability for practice patterns may be more productive in addressing inappropriate care.

\section{Methods}

\section{Data}

De-identified patient-level data about all public and private hospital separations (discharge, deaths and transfers) for the financial year 2010-11 were obtained from the Australian Institute of Health and Welfare after approval by each state and territory. The dataset included 8720771 records from 709 separate public hospital sites in all states except the Australian Capital Territory (private hospitals in each state were all grouped with a single code). Data were released as one record per admission, so it was not possible to link records to derive data on a per-person basis. Names of public hospitals were suppressed as part of the approval process.

Approval from an ethics committee was not required, but data confidentiality requirements were imposed as part of data release.

\section{Selecting treatments for assessment}

Potentially ineffective treatments were drawn from published lists of, or recommendations about, inappropriate care. These include a list of procedures identified as potential disinvestment candidates, ${ }^{9}$ a list of procedures where there had been a "reversal of evidence" that is, where subsequent evidence had shown that early treatment recommendations were no longer appropriate $^{10}$ — and examination of decisions of two national health technology assessment bodies: the Medical Services Advisory Committee in Australia and the

\section{Selection process for do-not-do treatments}

Exclude non-hospitalinpatient interventions $(n=516)$

Exclude advice with relatively weak or contested evidence (published prior to data period), or which was not supported by consulted clinical experts $(n=177)$

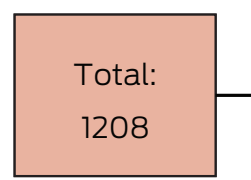
ambiguous about do-not-do status (eg, do-not-do "routinely") $(n=189)$

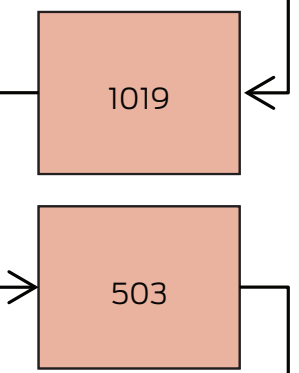

Exclude advice about patient groups, diagnoses or interventions that are not coded in our data (eg, pharmaceutical doses, un-coded tests) ( $n=317)$

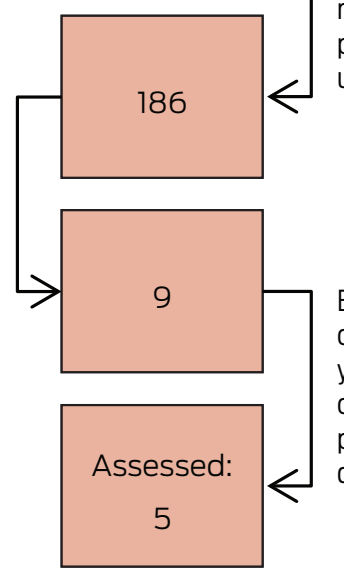

Exclude if there were five or fewer occurrences a year (not counting occurrences with a potentially legitimating diagnosis) $(n=4)$
Exclude advice that is 


\section{Potentially inappropriate procedures \\ Do-not-do \\ - Vertebroplasty for painful osteoporotic vertebral fractures ${ }^{11,12}$ \\ - Arthroscopic lavage or debridement for osteoarthritis of the knee ${ }^{13,14}$ \\ - Laparoscopic uterine nerve ablation for chronic pelvic pain ${ }^{15}$ \\ - Removing healthy ovaries during a hysterectomy ${ }^{16}$ \\ - Hyperbaric oxygen therapy for a range of conditions including osteomyelitis, cancer, non-diabetic wounds and ulcers, skin graft survival, Crohn's disease, tinnitus, Bell's palsy, soft tissue radionecrosis, cerebrovascular disease, peripheral obstructive arterial disease, sudden deafness and acoustic trauma, and carbon monoxide poisoning ${ }^{17-19}$ \\ Do-not-do-routinely \\ - Fundoplication to treat gastro-oesophageal reflux disease ${ }^{20}$ \\ - Episiotomy during spontaneous vaginal births without complications ${ }^{21}$ \\ - Amniotomy during normally progressing labour ${ }^{22}$}

National Institute for Health and Care Excellence in England. Only guidance published before our data period (2010-11) was used.

To select do-not-do treatments for analysis, these lists were whittled down by excluding recommendations that were vaguely expressed and treatments that were unable to be reliably coded using the International Classification of Diseases Australian Modification diagnosis and procedure codes or did not take place in hospitals. A further filter excluded treatments for which the evidence originally cited was weak or was contradicted by subsequent evidence, or was not supported by clinical experts we consulted. Finally, since we are investigating variation, we excluded cases with five or fewer occurrences and combined overlapping advice (Box 1). At each of these steps we took a conservative approach to reduce the chance that our analysis - a proof of concept - would be rejected based on the examples we used. We also looked at examples of treatments with recommendations against being performed "routinely", but these were selected opportunistically (as with do-not-do advice, the evidence was evaluated and clinical experts were consulted).

Do-not-do and do-not-do-routinely advice was expressed as "do not (routinely) do procedure $x$ for diagnosis $y^{\prime \prime}$. Clinical experts reviewed those cases which had multiple diagnoses listed to ensure that none of the additional diagnoses or procedures might provide a justification for the do-not-do (or do-not-do-routinely) procedure. Coding assignment of diagnoses and procedures was reviewed by an independent health information manager.

The filtering process excluded practically all of the original candidates of potentially inappropriate care. From the original lists, only five procedures were clearly potentially inappropriate and could be analysed in the data (Box 2). From the many do-not-do-routinely procedures, three were selected as exemplars.

\section{Measuring use of the selected treatments}

To look at variation in a fair and meaningful way, we measured the proportion of patients in a hospital who have the relevant diagnosis (such as osteoarthritis of the knee) who also received the do-not-do procedure (such as arthroscopic

3 Incidence of do-not-do treatments, 2010-11

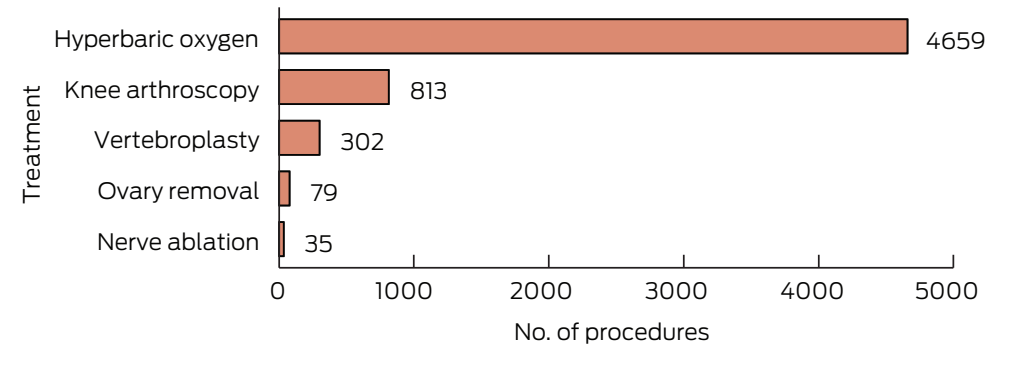

Among hospitals that perform the lavage or debridement). This partly addresses a criticism of the geographic research that it does not standardise adequately for differences in underlying rates of disease.

With this metric, we only compared hospitals that are able to provide the do-not-do treatment. These hospitals both perform the relevant procedure and treat patients $(>5)$ with the relevant morbidity.

\section{Results}

Incidence of each of the five do-not-do procedures is shown in Box 3.

The incidence of the five identified do-not-do treatments was quite low, with a total of 5888 procedures identified in the dataset. While this was a very small proportion of all patients, it is 4.5 per cent of all patients receiving the relevant procedures (or in one case, combination of procedures). It should be noted that this is a lower bound as we did not measure compliance with all do-not-do guidance for these procedures. relevant procedure and also treat patients $(>5)$ with the relevant morbidity, we found that the incidence of the do-not-do procedures was highly variable (Box 4).

For all the do-not-do treatments, the outliers with the highest rates were a long way from the average. There were 25 hospital departments that provided a do-not-do treatment more than three times as often as the average hospital (within hospital comparator groups). Eight hospital departments provided do-not-do treatments at over five times the 
average rate, while three departments did so at over 10 times the national rate.

Again, we saw substantial variability in hospital procedure rates for the procedures that should not be done routinely (Box 5), with some hospitals clear outliers. The hospitals with the highest rates for the three do-not-do-routinely treatments offered them at more than nine times, six times and double the average rate.

\section{Discussion}

This study is the first attempt to quantify the extent of inappropriate care in Australian hospitals for a range of conditions using routine data. We have shown that it is possible to use routine hospital data to identify the incidence of potentially inappropriate care.

Importantly, the procedures used here as examples have either been shown in academic studies to be inappropriate or are recommended against in guidelines, or both. What we have shown is that, despite this advice, and even defunding in the Medicare Benefits Schedule, the procedures are still being performed. Guidelines and funding policies are clearly not sufficient to solve this problem.

\section{Limitations}

This study has a number of limitations. First, it cannot be a basis for generalising about the overall incidence of inappropriate care in Australian hospitals. We used a small, non-representative sample of hospital procedures and analysed their incidence in a single year. Because inappropriate care appears to be relatively infrequent, there may be instability in the incidence of our indicator conditions.

Second, the inappropriate care identified in this study can only be considered potentially (rather than definitively) inappropriate. Some of the identified inappropriate treatments may be coding errors or may be justified on the basis of a rare combination of patient characteristics.

\section{Distribution of proportion of relevant patients receiving a do-not-do treatment in public hospitals with capacity to perform the do-not-} do treatment, 2010-11

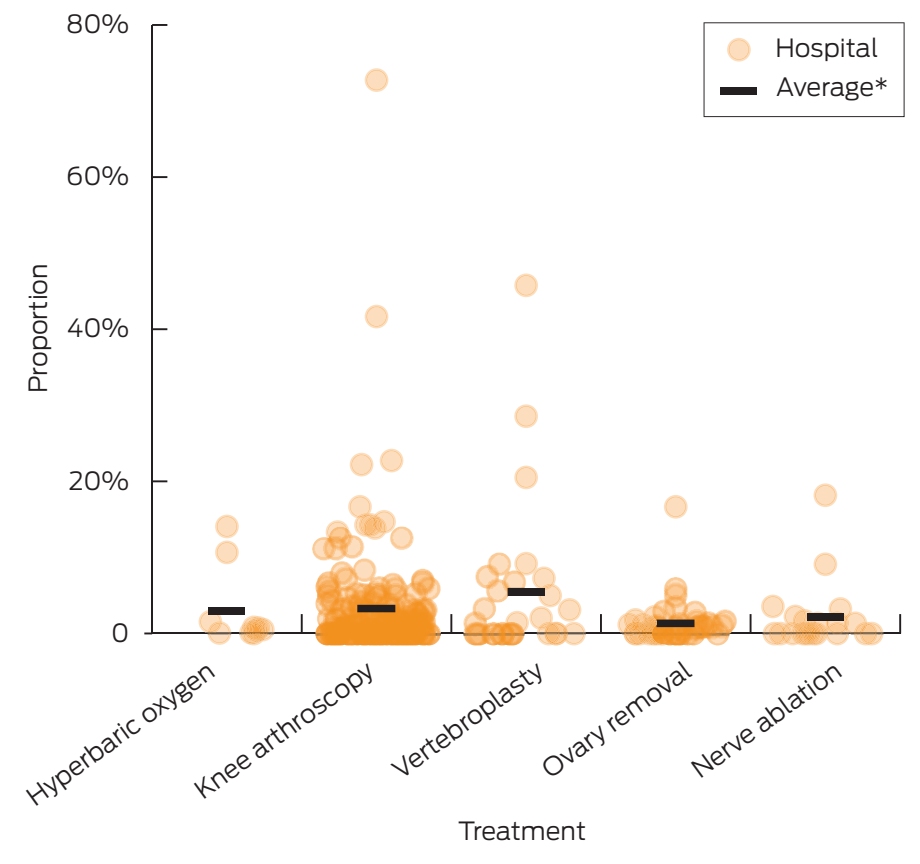

* Average rates among comparator hospitals: hyperbaric oxygen therapy (2.9\%); arthroscopic lavage or debridement of the knee (3.3\%); vertebroplasty $(5.4 \%)$; oophorectomy (1.4\%); uterine nerve ablation (2.1\%).

Routine data can only identify what is occurring, not why. We therefore suggest that the indicator be labelled "potentially inappropriate care", although the procedures themselves would remain indicators of, prima facie, inappropriate care.

Third, we were not able to analyse rates of inappropriate care in individual private hospitals.

Fourth, we were unable to link data across settings or time. A person who had multiple treatments, one of which was a do-not-do treatment, would thus be counted once in the numerator and multiple times in the denominator. This makes our prevalence estimates conservative.

\section{What should be done to ensure safe and effective care?}

Identifying potentially inappropriate practice is irrelevant unless action occurs. The steps outlined below will allow governments to monitor more and ultimately reduce inappropriate care in Australian hospitals.
First, forms of inappropriate care should be identified and communicated in a more consistent and accessible way. This should be a role for the Australian Commission on Safety and Quality in Health Care or the health productivity and performance commission foreshadowed in the 2014 federal Budget to take over functions from the Commission together with a number of other performance-related national authorities. Such a Commission should, with clinical involvement, identify additional procedures that represent potentially inappropriate care. It might draw on parallel efforts such as NPS MedicineWise's Choosing Wisely program and the Royal Australasian College of Physician's EVOLVE initiative. The Commission should maintain a centralised, easily accessible and continuously updated list of questionable procedures for clinicians to use.

Second, existing data should be used to measure and benchmark a wider range of inappropriate care. In this study, data access agreements meant we could only analyse a handful of 


\section{Distribution of proportion of patients receiving a do-not-do- routinely treatment in public hospitals with capacity to provide the treatment, 2010-11}

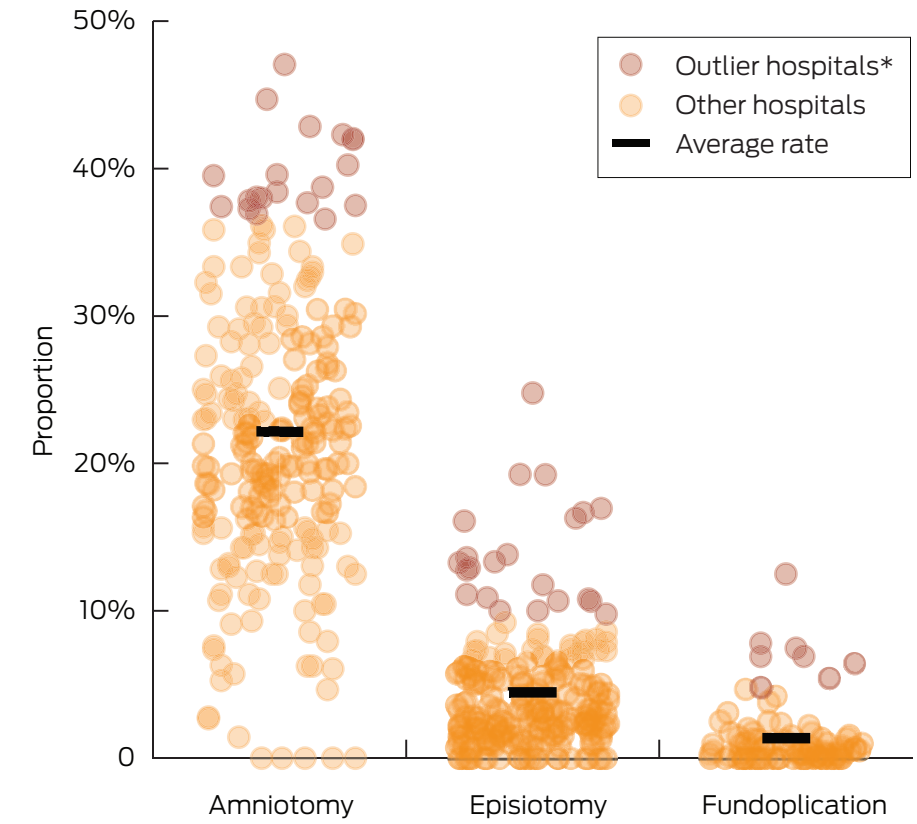

* The $10 \%$ of hospitals with the highest rates are identified as outliers.

do-not-do treatments. Many more can be analysed using additional sources of clinical guidance and data that the Australian government already possesses, and that the Commission should have access to. Studies of geographic variation have linked patient data across datasets and over time. ${ }^{23}$ Linkage of this kind could allow the Commission to do more; for example, analysing inappropriate use of treatments that should not be first-line interventions. It could also link patient records across different parts of the health system to cover hospitals, the Pharmaceutical Benefits Scheme and the Medicare Benefits Scheme.

Third, the Commission should advise states and hospitals about their rates of questionable care. States should give outlier hospitals a chance to improve, but if high rates persist there should be an external clinical review. The reviews should investigate all aspects of clinical decision making in the relevant department or specialist area. This would include confirming that the data accurately recorded the treatments that were chosen and assessing whether these choices were clinically valid.

States should prioritise investigation of hospitals with high rates of potentially inappropriate care, both for practical reasons and because low rates may reflect cases where most procedures were actually legitimate. States should also consider the volume of patients with the relevant morbidity. Our cut-off (> 5) could result in investigations that affect the care of very few people. Choosing thresholds that trigger investigations is ultimately a normative decision. It involves a trade-off of unnecessary investigatory burden and waste of resources against potential clinical risks to patients associated with inappropriate care.

Ultimately, patients should have a reasonable expectation of receiving appropriate care. Our study has shown that procedures that are contrary to contemporary clinical evidence are being done in Australia, and that some hospitals seem to provide a very high rate of these procedures. These hospitals should be alerted to the fact of their aberrant practice and be subject to clinical review if that practice continues, helping to ensure that hospital care in Australia is evidence-based, effective and safe.

Competing interests: No relevant disclosures.

(c) 2015 AMPCo Pty Ltd. Produced with Elsevier B.V. All rights reserved. 
1 Wennberg J, Gittelsohn A. Small area variations in health care delivery. Science 1973; 182: 1102-1108.

2 Wennberg JE. Forty years of unwarranted variation - and still counting. Health Policy 2014; 114: 1-2.

3 Organisation for Economic Cooperation and Development. Geographic variations in health care: what do we know and what can be done to improve health system performance? OECD Health Policy Studies. Paris: OECD Publishing, 2014.

4 Australian Commission on Safety and Quality in Health Care, Australian Institute of Health and Welfare. Exploring healthcare variation in Australia: analyses resulting from an OECD study. Sydney: ACSQHC, 2014.

5 Evans RG. The dog in the night-time: medical practice variations and health policy. In: Andersen TF, Mooney G, editors. The challenges of medical practice variations. London: MacMillan Press, 1990: 117-152.

6 Mercuri M, Gafni A. Medical practice variations: what the literature tells us (or does not) about what are warranted and unwarranted variations. J Eval Clin Pract 2011; 17: 671-677.

7 Goodman DC. Unwarranted variation in pediatric medical care. Pediatr Clin North Am 2009; 56: 745-755.

8 Elshaug AG, McWilliams JM, Landon BE. The value of low-value lists. JAMA 2013; 309: 775-776.

9 Elshaug AG, Watt AM, Mundy L, Willis CD. Over 150 potentially lowvalue health care practices: an Australian study. Med J Aust 2012; 197: 556-560. https://www.mja.com. au/journal/2012/197/10/over-150potentially-low-value-health-carepractices-australian-study

10 Prasad V, Vandross A, Toomey C, et al. A decade of reversal: an analysis of 146 contradicted medical practices. Mayo Clin Proc 2013; 88: 790-798.

11 Kallmes DF, Comstock BA, Heagerty $P J$, et al. A randomized trial of vertebroplasty for osteoporotic spinal fractures. N Engl J Med 2009; 36l: 569579.

12 Buchbinder R, Osborne RH, Ebeling $P R$, et al. A randomized trial of vertebroplasty for painful osteoporotic vertebral fractures. N Engl J Med 2009; 361: 557-568.

13 Moseley JB, O'Malley K, Petersen NJ, et al. A controlled trial of arthroscopic surgery for osteoarthritis of the knee. $N$ Engl J Med 2002; 347: 81-88.
14 Kirkley A, Birmingham TB, Litchfield RB, et al. A randomized trial of arthroscopic surgery for osteoarthritis of the knee. N Engl J Med 2008; 359: 1097-1107.

15 National Institute for Health and Clinical Excellence. Laparoscopic uterine nerve ablation (LUNA) for chronic pelvic pain. London: NICE, 2007. (NICE Interventional Procedure Guidance 234.)

16 National Institute for Health and Care Excellence. Heavy menstrual bleeding. London: NICE, 2007. (NICE Clinical Guideline 44.)

17 Medical Services Advisory Committee. Hyperbaric oxygen therapy. Canberra: MSAC, 2000. (MSAC applications 10181020. Assessment report.)

18 Medical Services Advisory Committee. 1054 - Hyperbaric oxygen therapy for the treatment of non-healing, refractory wounds in non-diabetic patients and refractory soft tissue radiation injuries. MSAC, 2003.

19 Medical Services Advisory Committee. Reconsideration of Application 1054.1: Hyperbaric oxygen treatment (HBOT) for non-diabetic chronic wounds. MSAC, 2012.

20 National Institute for Health and Care Excellence. Dyspepsia: management of dyspepsia in adults in primary care. (NICE Clinical Guideline 17.) London: NICE, 2004.

21 National Institute for Health and Care Excellence. Intrapartum care: care of healthy women and their babies during childbirth. (NICE Clinical Guideline 55.) London: NICE, 2007.

22 National Institute for Health and Care Excellence. Induction of labour. (Clinical Guideline 70.) London: NICE, 2008.

23 Schwartz AL, Landon BE, Elshaug $A G$, et al. Measuring low-value care in Medicare. JAMA Intern Med 2014; 174 : 1067-1076. 